\title{
A CLOSED CIRCUIT ELECTRO-HYDRAULIC ACTUATOR WITH ENERGY RECUPERATION CAPABILITY
}

\author{
Shaoyang $\mathrm{Qu}^{1 *}$, David Fassbender ${ }^{1}$, Andrea Vacca ${ }^{1}$, Busquets Enrique ${ }^{2}$, Uwe Neumann ${ }^{2}$ \\ ${ }^{I}$ Maha Fluid Power Research Center, Purdue University, Indiana, USA \\ ${ }^{2}$ Bosch Rexroth, South Carolina, USA \\ * Corresponding author: Tel.: +1 765409 3503; E-mail address: qu82@purdue.edu
}

\begin{abstract}
The recent electrification trend in the off-road market has incentivized research towards the proposal of compact, cost-effective and energy-efficient solutions for hydraulic actuators. As a result, increased attention has been given to electro-hydraulic actuator (EHA) architectures. The paper offers a study performed on a novel closed-circuit EHA architecture with the goal to maximize the overall system efficiency while meeting or exceeding traditional off-road applications performance, thereby enabling further electrification of off-road applications. Both numerical and experimental approaches are utilized to validate the functionality of the proposed EHA circuital configuration in four quadrants. Moreover, the actuator functionality at both high and low velocities are considered, which has never been explored in the past due to the limitations on the hydraulic machine driving speed. The good match between the experimental data and the simulation results confirms the potential of the simulation model for sizing such EHA architecture for different actuator sizes, duty cycles, and performance levels.
\end{abstract}

Keywords: Electro-Hydraulic Actuator, Closed Circuit, 4-Quadrant Functionality

\section{INTRODUCTION}

Nowadays, an increasing interest in replacing classical hydraulic architectures with energyefficient, compact and plug-and-play system configurations brings electro-hydraulic actuator (EHA) concepts to the center of the court. Hence, efforts aiming at the optimal solution for mobile applications never stop. However, apart from a few aerospace cases [1][2], EHAs have failed to penetrate the markets, especially construction and off-road vehicle markets, which contribute much to industrial energy consumption.

In terms of energy efficiency, an optimal hydraulic system should avoid metering losses and restore energy from the assistive load as much as possible. The core concept of EHAIndividualized actuator - makes the optimization achievable. The individualization levels of fluid power systems were scientifically investigated by Weber et al. [3], which clearly demonstrated the trade-off between cost and flexibility. In terms of solutions for variable EH units, a classification of different electro-hydraulic drive (EH) types has been studied [4][5], which comprises two options for EH units: one uses a variable electric motor and the other a variable pump. Studies on the fixed motor and variable pump (FMVP) configurations have shown good controllability [6]. Research on hydraulic transmission control based FMVP has also been conducted [7]. However, architectures with a variable motor and fixed pump (VMFP) receive more spotlight due to the better energy efficiency and cost consideration.

Based on VMFP, a compact EHA design has been obtained and proposed for its use in a 'more electric aircraft' [8], where the peak power of the unit is a constraint. A self-contained EHA with peak power scalable up to $80 \mathrm{~kW}$ has also been studied including a dynamic analysis [9]. Energy efficiency and performance analysis were conducted for a linear double drive EHA with self-locking function and scalable power up to 50 $\mathrm{kW}$ [10]. As for energy regeneration and recuperation, relating to applications on hydraulic excavators, studies have demonstrated large regenerative power sections for a boom actuator and presented a possible solution including an accumulator-motor-generator [11]. 
A novel driving system with an EHA concept for hydraulic excavators has been proposed using an asymmetric pump, which is able to regenerate more than $80 \%$ of potential energy [12].

One of the most significant technical challenges impeding the introduction of industrialized EHAs is the pump speed limitation constraint, which hinders the functionality of closed-circuit EHAs for low-speed actuation. This paper addresses this challenge and proposes a solution. The study presented in this paper is structured in the following order: system configuration of proposed EHA, accumulator sizing for the self-contained system, development of simulation model and results, experimental setup and test results, conclusion and outlook.

\section{SYSTEM CONFIGURATION}

\subsection{Hydraulic architecture}

The proposed system configuration is shown in Figure 2. A fixed-displacement hydraulic pump (HP) and a variable speed electric motor (EM) constitute the individual EH drive unit. The actuator is a single-rod double-acting cylinder (CYL). The differential flow required by the difference between the bore side and rod side area is compensated by the low-pressure accumulator (ACC), together with two pilot check valves
(PCV) controlling its charging or discharging. Two directional on/off valves (DV) allow for load holding at rest condition and the flow direction that realizes the cylinder motion is dictated by the four-quadrant EH unit. Pressure relief valves (RV) are installed on both sides of the pump in order to avoid over-pressurizations in either extension or retraction. A proportional $2 / 2$ directional valve is used as a bypass (BP) parallel to the cylinder, which allows for slow or fast actuation when exceeding the speed range of the gear pump. This will be further explained in the following sections. A filter (FL) and two check valves (CV) are necessary for a selfcontained implementation. An external pump drain line needs to be connected to the accumulator to maintain the overall amount of fluid within the system constant for all possible CYL positions.

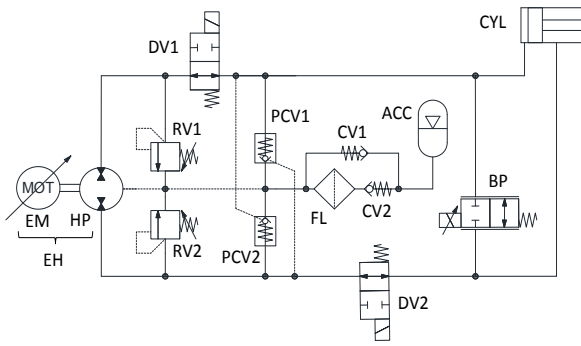

Figure 2: Proposed system configuration

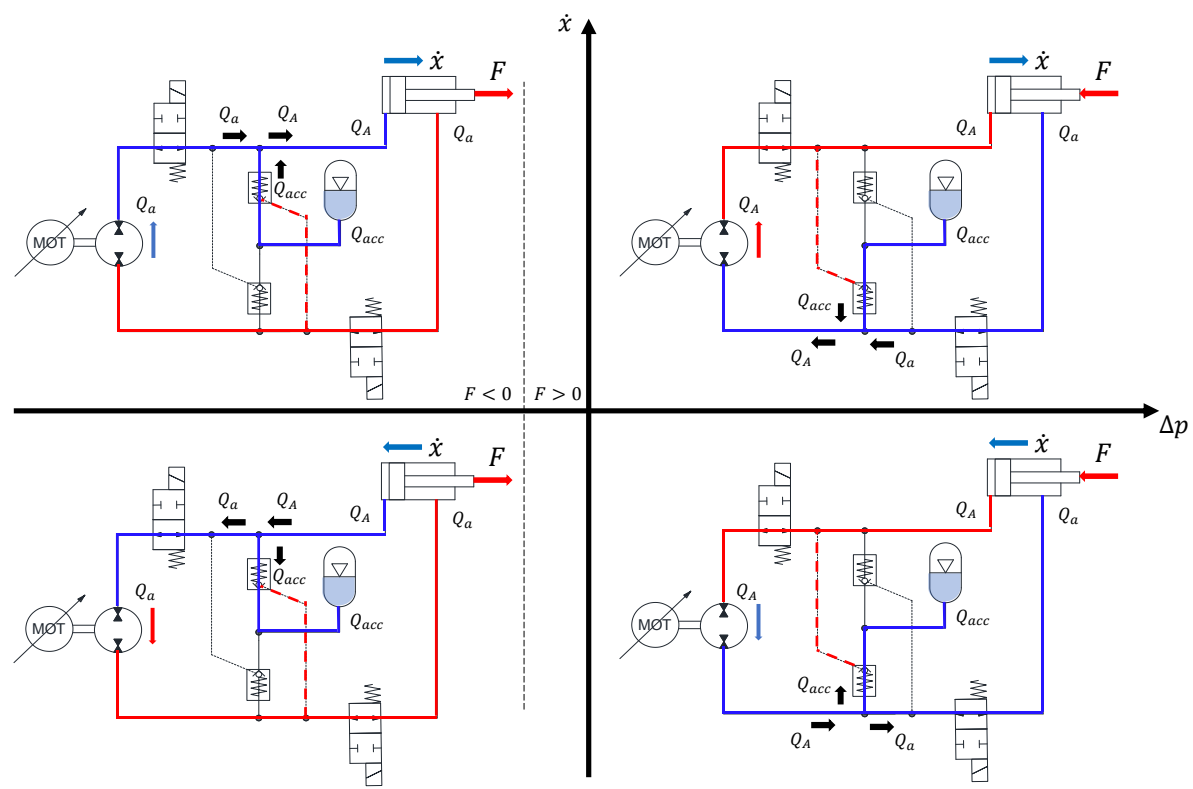

Figure 1: Functionality in four quadrants illustrated by simplified schematic 


\subsection{Four-quadrant functionality}

The functionality of the proposed architecture can be summarized with the representation of Figure 1, where the schematic is simplified for a clear representation of the operating conditions in 4 quadrants. The $\mathrm{x}$-axis shows the pressure difference between the cylinder chambers, which is positive for higher pressure on the piston side. The $y$-axis shows the actuator velocity. A positive velocity means the extension of the cylinder. High/low pressures are highlighted by red/blue color. Because of the cylinder differential area, the corresponding port flow rates are different, which are denoted as $Q_{A}$ and $Q_{a}$. On the contrary, the pump inlet and outlet are ideally constant. Therefore, the differential flow needs to be compensated by the flow from/to accumulator, as $Q_{a c c}=Q_{A}-Q_{a}$. It follows that the accumulator must always discharge in the extension phase and charge in the retraction phase. In summary, the first and third quadrants outline the pumping modes of the EH unit. The motoring modes on the other hand are given in the second and fourth quadrants, where the overrunning load makes it possible to recuperate energy by accumulator and electronic subsystems for storing energy. Since the accumulator defines the lowest pressure in the circuit, the DVs are located outside of the discharging path of $\mathrm{ACC}$, in order to decrease the possibility of cavitation.

It should be noted that in the four-quadrant map, the origin represents an equal pressure in both chambers of the cylinder and no motion, but not a zero load $F$ due to the differential cylinder areas. A line representing zero load is noted across the second and third quadrant in Figure 1. It is also important to note that a switch between modes may pose challenges on the stable motion of the actuator [13].

\subsection{Modes beyond speed range of $\mathrm{EH}$}

Even though the functionality shown in Figure 1 may cover most hydraulic applications, the operating conditions beyond the speed limitation of the EH unit are not considered. Slow cylinder speeds are challenging as common fixed displacement pumps typically cannot be operated below a certain minimum shaft speed. The idea of the proposed solution consists of dividing the flow into the circuit by using BP. Focusing on the fourth quadrant in Figure 1 for the retraction phase with overrunning load, an illustration with more details is given in Figure 3, which highlights the function of BP.

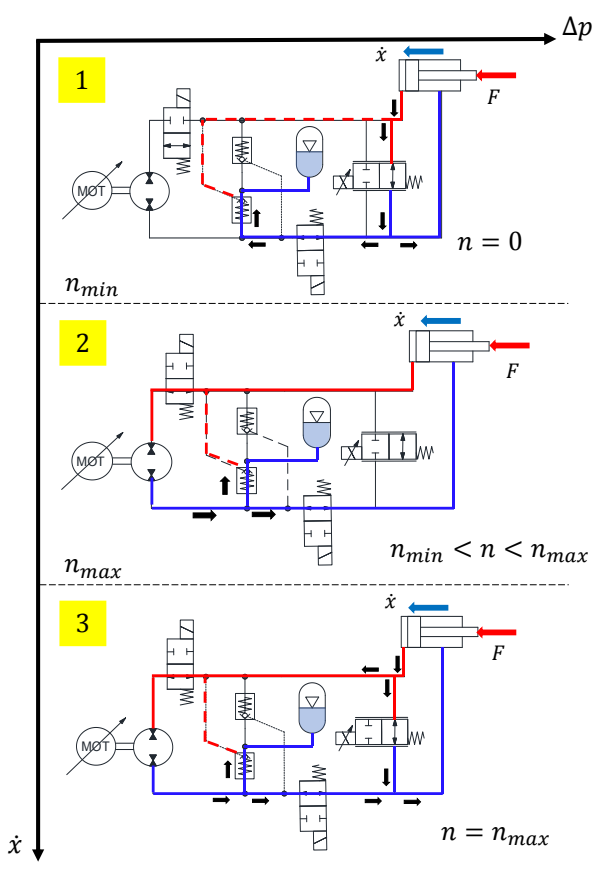

Figure 3: Working modes in the retraction phase with overrunning load

The y-axis shows the actuation speed, which is in normal mode proportional to the speed of the EH unit $\left(n_{\min }<n<n_{\max }\right)$. This condition is given in the middle - case 2. However, the EH unit cannot handle the low speed under $n_{\min }$. As illustrated in case 1, the bypass valve controls the low-speed retraction without the pump. The BP works as a metering orifice, and the differential flow still comes from the low-pressure accumulator. Although an overrunning load is applied, different from case 2, the EH unit will not recuperate energy for case 1 . Furthermore, a high-speed mode may be required in certain applications where the flow demand is higher than the maximum limit of the EH unit. Case 3 represents this high-speed condition. Both $\mathrm{EH}$ and $\mathrm{BP}$ are activated and determine the retracting speed. Ideally, a maximum speed is set at the EH unit, as given in case 3 , to avoid an excess of flow going though BP resulting in metering losses. The solution for covering low/high speeds works not only in the second quadrant as described in this paper, but also for all others quadrants. 


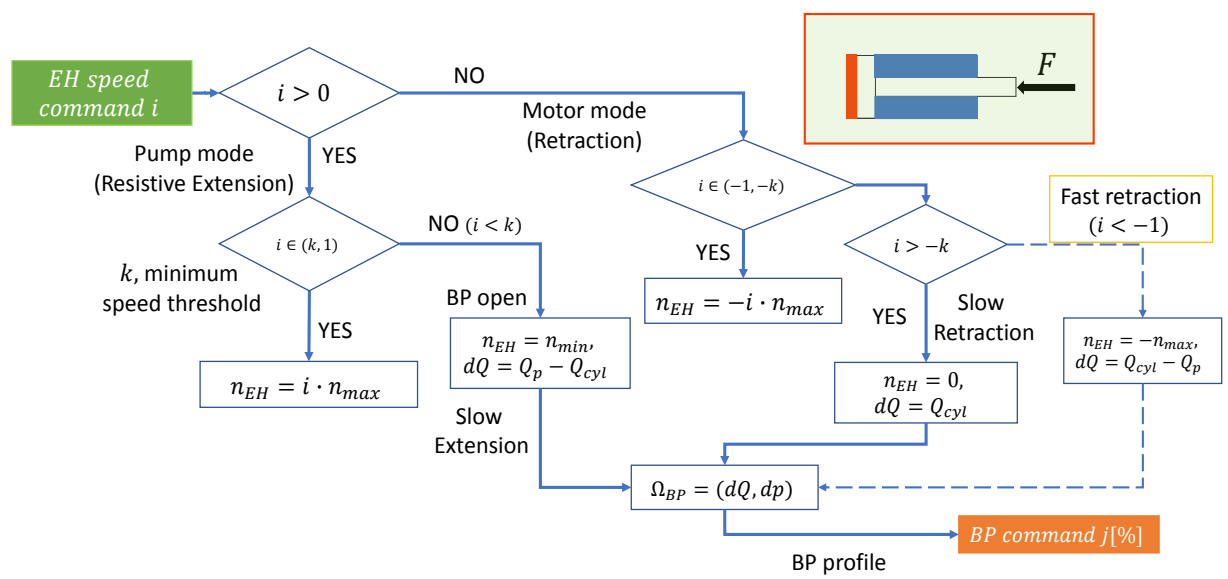

Figure 4: Conceptual flow chart for logic of switching modes

Details are omitted for brevity. Essentially, the first and third quadrants are symmetric and have the same modes, while the second and fourth share the same modes of Figure 3.

\subsection{Logic for switching modes}

In order to control the actuation velocity, two inputs are applied to the system: the speed command to the EH unit and the command to control the opening of BP. A third signal to open the DVs is always given, when any speed is demanded. The logic for determining these commands is summarized in Figure 4, covering all cases where the load on the cylinder is positive (first and fourth quadrants in Figure 1). A typical case for this working mode can be represented by a gravitational load, such as the boom implement of a construction machine, or the hitch of an agricultural tractor.

The operator gives a certain speed command $i$ which is converted to the pump speed and control current of BP. Then, $i$ can be easily defined as a normalized number with a range between -1 and 1. Two modes are distinguished first—pumping mode (resistive, quadrant 1) and motoring mode (assistive, quadrant 4) - by the sign of the given command. The command compared to a threshold $k$ defines the next sub-model in order to keep the pump speed above the minimum limit. If the magnitude of $i$ is greater than $k$, BP does not need to be opened, and the pump can control the actuation speed itself. Slow modes including extension and retraction are activated if the magnitude of $i$ is less than $k$. For slow extension, the desired actuator flow rate $Q_{c y l}$ is obtained by the difference between pump flow $Q_{p}$ and the flow rate of $\mathrm{BP}(d Q)$. On the other hand, under the slow retraction case the pump speed is set to zero, and the actuator flow is only controlled by BP. To control the opening of BP, the flow rate $d Q$ as well as the pressure difference $d p$ are needed, resulting in a requirement of pressure sensors(as listed in the experimental section, Table 2). An exception-mode $i$ less than -1 is denoted with a dotted line in the flow chart. While the pump already operates at maximum speed, an extra flow from the actuator circulates through BP. At the end, by knowing the $(Q, d p)$ characteristics of $\mathrm{BP}$, it is possible to determine the command $j$ for the desired opening area.

\section{SIZING OF ACCUMULATOR}

The ACC has two essential roles in the selfcontained EHA system: 1) as a flow source and 2) as a reservoir, allowing the CYL differential flow requirement to be compensated. The drain line of the pump is also connected to the ACC in order to avoid losing flow during operation. Moreover, the ACC energy can be recuperated during overrunning phases and introduced back into the system during resistive phases of the CYL motion. These aspects have to be taken into account when sizing ACC.

In general, a hydro-pneumatic accumulator utilizes the compressibility of gas to store energy and adjust flow in a hydraulic circuit. According 
to the ideal-gas law (assuming nitrogen as an ideal gas) the relationship between gas or oil volume and pressure of the accumulator can be generalized as a polytropic process:

$p V^{n}=p_{0} V_{0}^{n}=\mathrm{C}$

For an adiabatic process, $\mathrm{n}$ is equal to 1.4. According to the equation, Figure 5 represents the relationship between volume and pressure of the gas inside the ACC. The pre-charge status is $\left(p_{0}, V_{0}\right)$, where $V_{0}$ is the ACC total volume. Two states $\left(p_{1}, V_{1}\right)$ and $\left(p_{2}, V_{2}\right)$ correspond to both ends of the cylinder stroke.

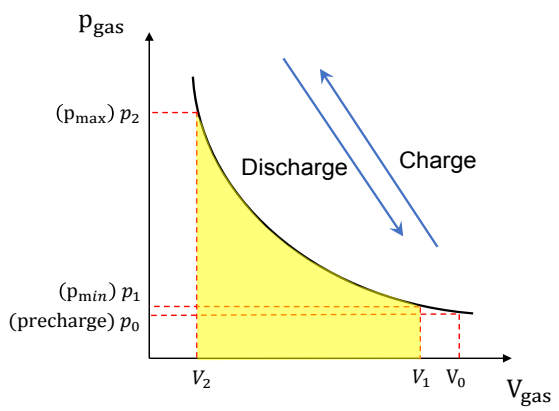

Figure 5: Polytropic process of gas in the accumulator The condition $p_{0} / p_{1}=90 \%$ ensures that a minimum amount of oil always remains in the ACC which can compensate losses due to leakage across the cylinder rod seal. The volume to compensate the differential flow is given by:

$\Delta V=V_{1}-V_{2}=V_{\text {rod }}=\frac{\pi d^{2} L}{4}$

With respect to the pump drain line restrictions $p_{2}<p_{\text {drain }}$ is a further restriction, which leads to:

$p_{2}=p_{0}\left(\frac{V_{0}}{\sqrt[n]{0.9} V_{0}-V_{\text {rod }}}\right)^{n}<p_{\text {drain }}$

For a maximum drain line pressure of $10 \mathrm{bar}$ and the consideration of compactness, the size of $\mathrm{ACC}$ is chosen as $4 \mathrm{~L}$ with a pre-charge pressure of $4.5 \mathrm{bar}$.

\section{SIMULATION MODEL}

\subsection{Parameters}

Based on the configuration proposed in Figure 2, a lumped parameter simulation model of the proposed EHA was built in Simcenter Amesim (Simens PLM Software). For the purpose of conducting an initial validation of the system functionality, the hydraulic components are developed with basic equations, as outlined in Table 1. To extend the accuracy of the model, actual performance data for the individual components is included in the model.

Table 1: Development of the simulation model
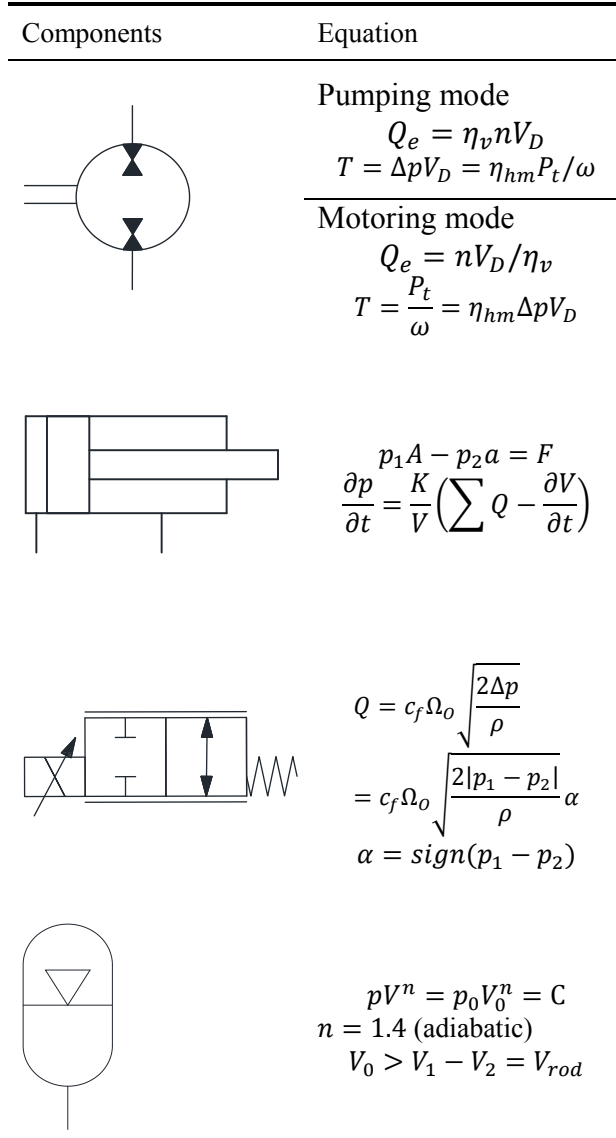

$$
\begin{gathered}
p V^{n}=p_{0} V_{0}^{n}=\mathrm{C} \\
n=1.4 \text { (adiabatic) } \\
V_{0}>V_{1}-V_{2}=V_{\text {rod }}
\end{gathered}
$$

The parameter setting of the simulation model considers the components chosen and utilized in the experimental setup, which will be described in Chapter 5. The main parameters of the model are summarized in Table 2.

Table 2: Main parameters in the simulation model

\begin{tabular}{lc}
\hline Parameter & Value[unit] \\
\hline Length of stroke $L$ & $0.8865[\mathrm{~m}]$ \\
Diameter of piston $D$ & $69.9[\mathrm{~mm}]$ \\
Diameter of rod $d$ & $44.2[\mathrm{~mm}]$ \\
Pump displacement $V_{p}$ & $14.53[\mathrm{cc} / \mathrm{rec}]$
\end{tabular}




\begin{tabular}{lc} 
Volume of Accumulator $V_{0}$ & $4[\mathrm{~L}]$ \\
Pre-charge pressure $p_{0}$ & $4.5[\mathrm{bar}]$ \\
Pump maximum speed $n_{\max }$ & $4000[\mathrm{rpm}]$ \\
Pump minimum speed $n_{\min }$ & $600[\mathrm{rpm}]$ \\
Relief pressure $p_{r}$ & $210[\mathrm{bar}]$ \\
\hline
\end{tabular}

\subsection{Simulation results}

Based on the given parameters and the proposed system architecture, simulations are conducted to validate the different modes' functionality in steady-state. To do so, an input command is given to the system each time. This results in an actuation velocity and pump speed as outputs. All working conditions in the four-quadrant map are covered, including extension and retraction with overrunning or resistive loads.

A force pushing the cylinder is defined as positive (first and fourth quadrants in Figure 1), while negative force is pulling (second and third quadrants in Figure 1). The direction of extension is defined as positive for the velocity. For positive forces up to $70 \mathrm{kN}$, the functionality is represented in Figure 6. The volumetric efficiency of pump is simplified as $100 \%$ ideally, and HP speed only depends on the command. Moreover, the CYL velocity must be robust with respect to different load conditions. This is reasonable as the behavior of the EHA should be independent of the load. The area ratio of the single-rod cylinder results in the different velocities for extension and retraction, as the same flow rate is going into the actuator. During fast assistive retraction, the pump is set to maximum speed, and BP is opened at a certain rate to achieve the same velocity as under the resistive retraction. During slow CYL motions, the pump speed is set at the minimum speed in the resistive phase, while with assistive loads the EH needs to be set to zero speed. A mismatch can be noticed for a negative full command. The reason is that the simplified control does not implement the exact valve characteristics of BP for all possible pressure drops.

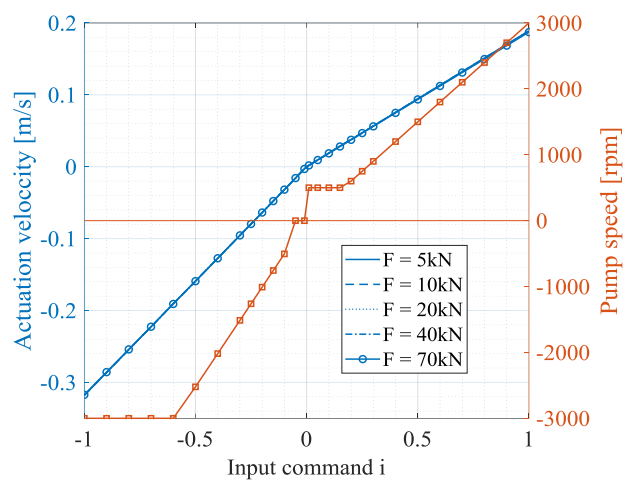

Figure 6: Simulated working modes in the first \& fourth quadrants

For negative loads, the results look different, as shown in Figure 7. Because of the specifications of the EHA, at the same actuation velocity, the pump speed is different from the actuation at a positive load. For instance, in assistive extension modes, pump flow is determined by the CYL rod side, noted as $Q_{a}$ in the second quadrant in Figure 1. In this case, the maximum pump speed is not required for the maximum demanded actuator velocity. On the other hand, retraction becomes resistive, so that the maximum retraction speed leads to the saturation given in Figure 6, where BP is opend as explained. Another important fact caused by the area ratio is that the origin of zero force on the cylinder interface does not match zero pressure difference between both sides of the pump. For the $0 \mathrm{kN}$ loading case, the pressures in the cylinder chambers are different. Though not significant, the pressure in the CYL rod side is higher than the piston side. As a result, the EH unit operates in a resistive pumping mode with cylinder extension, while it operates as a motor when retracting the cylinder. When it comes to the $\mathrm{EH}$ unit, the $0 \mathrm{kN}$ case is similar to a negative loading cases. This case is denoted in the second and third quadrants in Figure 1. Figure 7 shows the results of negative and zero loading conditions.

It can be seen that the curve in the slow velocity mode area is not linear. According to the logic represented in Figure 4, if the load is resistive, the pump is set to minimum speed, otherwise to zero, and the activated BP controls the actuation. 


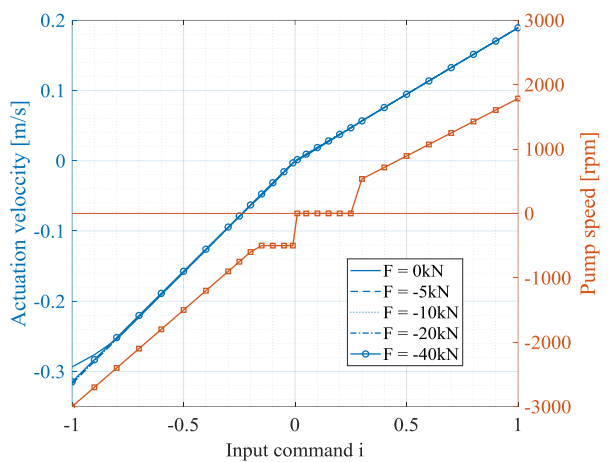

Figure 7: Working modes in second \& third quadrants

For a better understanding of the low-speed modes, Figure 8 represents a comparison between two typical conditions, based on normalized actuation velocity and EH speed. 20 $\mathrm{kN}$ force is applied to the cylinder, in both directions. In theory, the actuation velocity should closely follow the command, $(10 \%$ command meaning $10 \%$ of the maximum speed). The maximum command $(i=1)$ should result in the maximum speed in resistive modes. As explained before, the pump works in different modes and does not follow the input command in all modes, which can be also observed in Figure 8. Two factors dominate the pump speed in the EHA. Firstly, the same extension/retraction speed may require different flow rates from the pump, according to the loading condition, which can be assistive or resistive. The impact can be well seen for input commands higher than 0.3 in Figure 8. Secondly, BP is opened under slow modes (zoomed-in part in Figure 8) and fast retraction modes (command $i<-0.5$ ), so that the pump flow can be divided and re-circulated in the circuit. During slow motions, the pump speed is set to minimum speed in resistive phase, while under assistive loads it is zero. As given in the zoomed-in part of Figure 8, the slow modes for positive and negative loads are symmetric around origin, while the actuation velocity remains linear with the input command.

\section{EXPERIMENTS}

\subsection{Test rig setup}

A test rig is built at Maha Fluid Power Research Center, Purdue University, Lafayette, USA to validate the functionality of the proposed system. The conceptual structure of the test rig is shown in Figure 9.

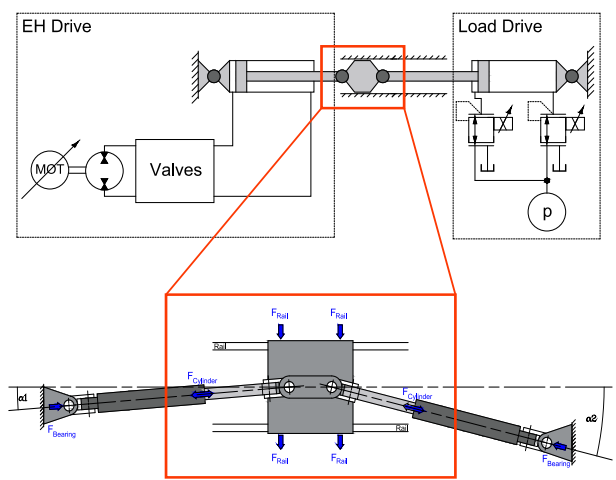

Figure 9: Conceptual structure of the test rig

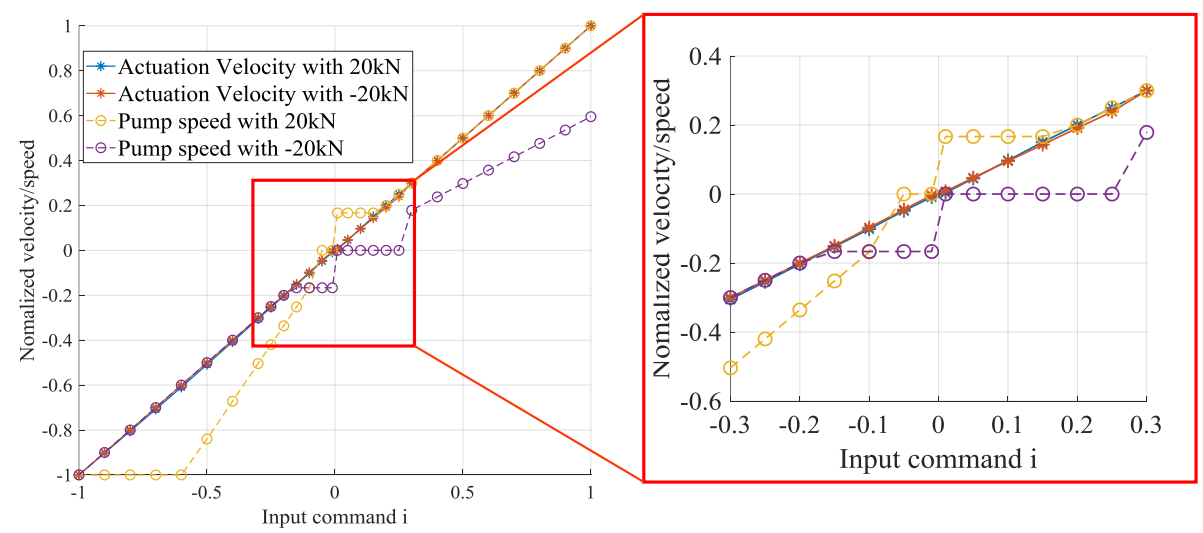

Figure 8: Actuation velocity/pump speed vs input command $i$ from simulation 
The designed test rig consists of two coupled cylinders. One cylinder is operated as part of the proposed EHA system while the other cylinder is pressure controlled and can be used to apply a defined load force to the EH drive. The cylinders are on both sides connected by dual-axis joints which prevent bending forces on the cylinder in case of misalignment. Side forces resulting from misalignment are compensated by linear ball rail guides.

The electric hardware needed for control and data acquisition on the test rig is listed in Table 3, including electronical control units, sensors, valves, and a servomotor.

Table 3: Hardware list for test rig

\begin{tabular}{lc}
\hline Hardware & Product number \\
\hline Controller & NI cRio-9024 \\
Analog modules & NI9264 NI9205 \\
& NI9472 \\
Digital modules & NI9403 NI6421 \\
& NI9472 \\
Pressure sensor 600bar & Rexroth R917A10105 \\
Position sensor 1000mm & ASM WS42 \\
On/off valves & Rexroth \\
& OD150536A000000 \\
Prop. 2/2 directional valve & KKDSR1NB/HCG24N \\
& 0K4V \\
Gear pump/motor & Rexroth \\
HDP servomotor & AZMF - 12 -016 URR \\
& 12 ML \\
Inverter & ABB \\
\hline The & ABB ACS800-U11 \\
\hline
\end{tabular}

The platform for control and data acquisition was established between hardware (sensors, NI cRio) and software (Simulink, Veristand).

The experimental setup located in the Maha Lab can be seen in the picture in Figure 10.

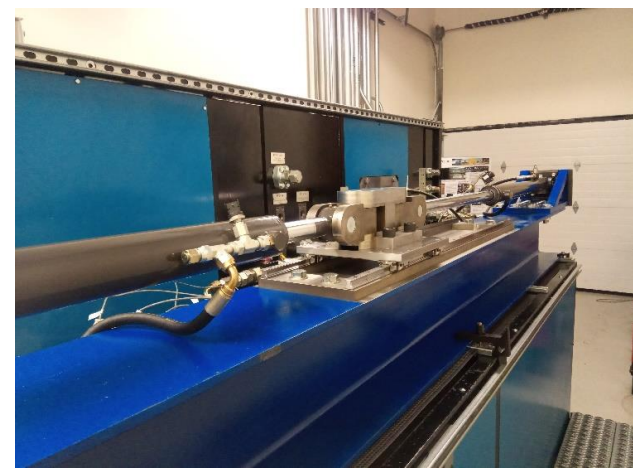

Figure 10: Experiment set up of the proposed EHA

\subsection{Measurements}

Measurements are conducted in order to show the functionality of the proposed circuit and to validate the results from the simulation. Input commands are the same for the experiments as for the simulation. Differently to the simulation, actuation velocities do not stay exactly the same at different loading conditions. Figure 11 represents an example of that.

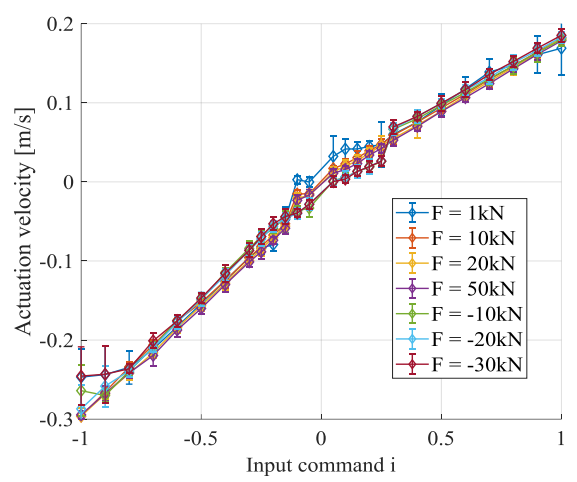

Figure 11: Measured actuation velocity from the test

One reason is that the actual pump has an efficiency map, meaning the volumetric efficiency may vary with different loading conditions. In addition, the low-speed mode works but the behavior is not as linear as desired for low speeds close to zero due to the nonlinear characteristics of $\mathrm{BP}$ under this range. More tuning of the parameters of BP may help to improve the performance. Moreover, fast retraction in resistive phases (minus force) may cause cavitation due to DV1 given in Figure 2.

For validation, a comparison between simulation and measurements is made based on normalized 
velocity/speed. Figure 12 shows the results at a $20 \mathrm{kN}$ load, which represents the baseline in Figure 6 from the simulation.

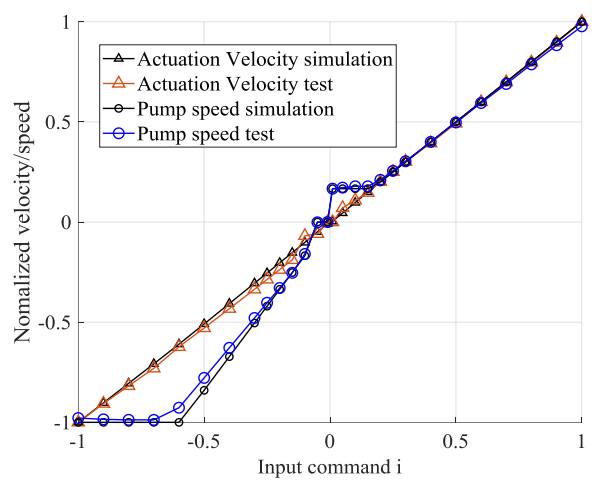

Figure 12: Comparison between measurements and simulation, $\mathrm{F}=20 \mathrm{kN}$

The results match well as given in the figure, though a minor difference is noticeable between the actuation velocity in the test and the simulation. Since the EH unit developed in the simulation model is always assumed ideally $100 \%$ efficient, it is reasonable that in the test extension can be slightly slower, while retracting slightly faster.

As for the cases given in Figure 7, a negative $20 \mathrm{kN}$ load is applied as well. The results are shown in Figure 13. The slow mode works as expected, and the linear performance can be improved by further control strategies on BP. As all measured points in the figure are actually the average of a certain time-period measurement, the noticeable discrepancies can be caused by measuring errors and noise.

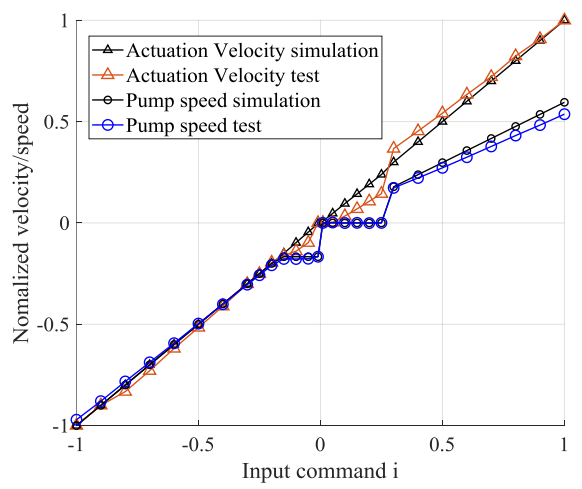

Figure 13: Comparison between measurements and simulation, $\mathrm{F}=-20 \mathrm{kN}$
In summary, all working modes in all fourquadrants are reflected in the test, and the functionality of the proposed novel EHA system was proved. An optimized control structure and efficiency map of EH may help improve the accuracy and to match the results between simulation and measurements.

\section{CONCLUSION AND OUTLOOK}

This paper presented a novel closed-loop EHA system, with the capability to cover a broad range of velocities and to recuperate energy. Following both numerical and experimental approaches, the performance verification, as well as the validation of its functionality in four quadrants was conducted for the proposed EHA. The method of sizing the accumulator was described, as a design guideline for different actuator sizes, duty cycles, and power levels. With the utilization of a bypass valve in the closed circuit, slow actuation speeds can be achieved by means of proper control logic for switching between high and low-speed control modes. Based on an experimental set up developed at Maha Fluid Power Research Center, all possible modes to operate the EHA were tested. The performance of experiments at a steady-state was compared with the simulation results obtained from the Amesim model. The good match between experiments and simulation confirms the potential for further applications for such kind of EHA architecture. With the basis of solid functionality, more research can be conducted in the future on the dynamic parts, such as an optimized control strategy for EHA systems. In addition, the energy-saving capability of the proposed EHA can be focused on more details. Especially a comparison with classical hydraulics is needed to study potential energy benefits and efficiency improvements. Moreover, long-term tests to validate the self-contained performance will be done. Ongoing studies also focus on the thermal behavior of closed-loop EHAs. Finally, solutions for cooling and filtration must be investigated in order to enable the technical realization of EHAs in commercial high-power applications.

\section{NOMENCLATURE}

$\begin{array}{ll}\text { EHA } & \text { Electro-Hydraulic Actuator } \\ \text { EH } & \text { Electro-Hydraulic Drive } \\ \text { FMVP } & \text { Fixed-Motor-Variable-Pump }\end{array}$ 


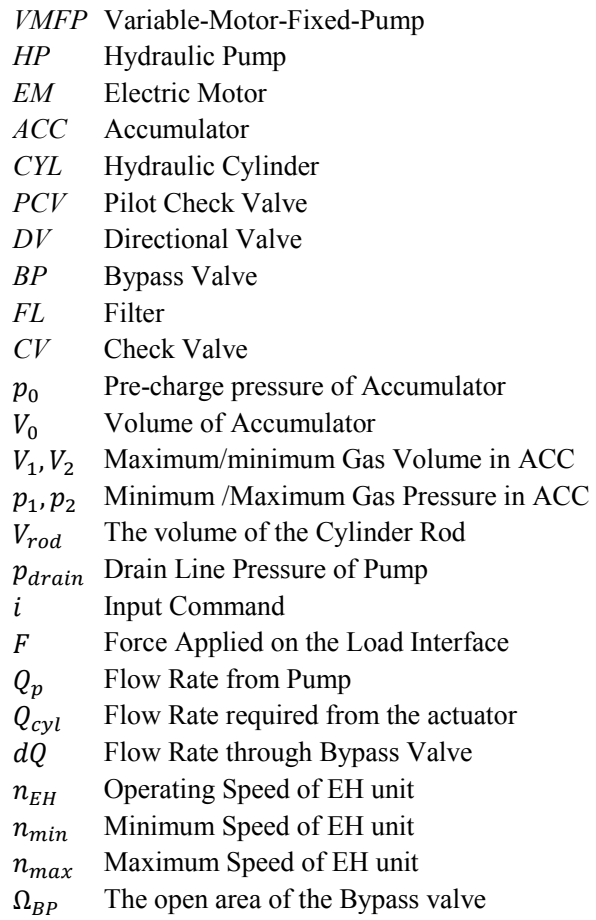

\section{REFERENCES}

[1] Navarro R (1997) Performance of an electrohydrostatic actuator on the F-18 systems research aircraft. NASA Tech Memo

[2] Frischemeier S (1997) Electrohydrostatic actuators for aircraft primary flight controltypes, modelling and evaluation. Proc Fifth Scand ...1-16. https://doi.org/10.15480/882.236

[3] Beck DB, Fischer DE, Kolks DG, et al (2016) Novel System Architectures by Individual Drives. 10th Int Fluid Power Conf 29-62

[4] Mattos W, Brazil BR (2016) 4EE - High Performance Energy Efficiency for Hydraulic Machine Drive 4EE Energy Efficiency
[5] Ketelsen S, Padovani D, Andersen TO, et al (2019) Classification and review of pumpcontrolled differential cylinder drives. Energies 12:1-26. https://doi.org/10.3390/en12071293

[6] Habibi S, Goldenberg A (1999) Design of a New High Performance. Int Conf Adv Intell Mechattronics 227-232

[7] Pastrakuljic V (1995) Design and Modeling of a New Eleciro Hydraulic Actuator. 137

[8] Altare G, Vacca A (2015) A Design Solution for Efficient and Compact Electro-hydraulic Actuators. Procedia Eng 106:8-16. https://doi.org/10.1016/j.proeng.2015.06.003

[9] Padovani D, Ketelsen S, Hagen D, Schmidt L (2019) A self-contained electro-hydraulic cylinder with passive load-holding capability. Energies 12:1-19. https://doi.org/10.3390/en12020292

[10] Schmidt L, Ketelsen S, Brask MH, Mortensen KA (2019) A class of energy efficient selfcontained electro-hydraulic drives with selflocking capability. Energies 12:1-27. https://doi.org/10.3390/en12101866

[11] Lin T, Wang Q (2012) Hydraulic accumulatormotor-generator energy regeneration system for a hybrid hydraulic excavator. Chinese J Mech Eng (English Ed 25:1121-1129. https://doi.org/10.3901/CJME.2012.06.1121

[12] Ge L, Quan L, Li Y, et al (2018) A novel hydraulic excavator boom driving system with high efficiency and potential energy regeneration capability. Energy Convers Manag 166:308-317.

https://doi.org/10.1016/j.enconman.2018.04.04 6

[13] Williamson C, Ivantysynova M (2010) Stability and Motion Control of Inertial Loads with Displacement Controlled Hydraulic Actuators. 6th FPNI - PhD Symp 499-514 